\title{
Incorporating genomics into breast and prostate cancer screening: assessing the implications
}

\author{
Susmita Chowdhury, MD, MPhil ${ }^{1}$, Tom Dent, MB, DPH ${ }^{1}$, Nora Pashayan, MD, PhD², \\ Alison Hall, BA, MA ${ }^{1}$, Georgios Lyratzopoulos, MD, FRCP ${ }^{3}$, Nina Hallowell, MA, DPhil'1, \\ Per Hall, MD, PhD ${ }^{4}$, Paul Pharoah, BM BCh, $\mathrm{PhD}^{3,5}$ and Hilary Burton, BM BCh, FFPHM ${ }^{1}$
}

Individual risk prediction and stratification based on polygenic profiling may be useful in disease prevention. Risk-stratified population screening based on multiple factors including a polygenic risk profile has the potential to be more efficient than age-stratified screening. In this article, we summarize the implications of personalized screening for breast and prostate cancers. We report the opinions of multidisciplinary international experts who have explored the scientific, ethical, and logistical aspects of stratified screening. We have identified (i) the need to recognize the benefits and harms of personalized screening as compared with existing screening methods, (ii) that the use of genetic data highlights complex ethical issues including discrimination against high-risk individuals by insurers and employers and patient autonomy in relation to genetic testing of minors, (iii) the need for transparency and clear communication about risk scores, about harms and benefits, and about reasons for inclusion and exclusion from the risk-based screening process, and (iv) the need to develop new professional competences and to assess cost-effectiveness and acceptability of stratified screening programs before implementation. We conclude that health professionals and stakeholders need to consider the implications of incorporating genetic information in intervention strategies for health-care planning in the future.

Genet Med 2013:15(6):423-432

Key Words: cancer; COGS; genetic information; implications; personalized; prevention; public health; risk; screening; stratification

\section{INTRODUCTION}

Increased knowledge about the genetic basis of cancer may enable more personalized health care for cancer ${ }^{1}$ that is tailored to the characteristics of the individual rather than the average population. Although more attention has been paid to the implications of this development for treatment, it is likely also to change the way preventive strategies such as screening are arranged, with screening interventions adjusted to reflect each person's level of risk. Such risk-stratified population disease prevention would have important implications for how screening is organized, how the generated information is handled, and the ethical, legal, and social issues that are raised. Although there is a growing body of literature about the potential clinical utility of genomic risk-stratified screening, ${ }^{2-4}$ there is little published analysis of the public health implications of population disease prevention strategies that use genomics.

How would stratified screening work? Genome-wide association studies have identified susceptibility variants that are common in the population, but each variant confers only a small increase in disease risk. ${ }^{5,6}$ Studies have reported that the addition of risk information from these common susceptibility variants to established risk models, such as those of Gail or Tyrer-Cruzick, may lead to better discrimination, ${ }^{7-10}$ that is, they may improve the ability of the model to correctly rank the degree of risk in an individual relative to that of the population as a whole. However, this is not enough. To be suitable for use, a risk-stratification model must have adequate discrimination and calibration (accurately estimating the population's average risk); it must also have clinical utility, which includes producing risk distributions for categories of people that are separated widely enough to justify different management of each category and so as to improve outcomes overall. Many risk alleles acting together may result in a distribution of risk in the population wide enough to be clinically useful for population stratification. ${ }^{11}$ Whether polygenic models will in fact satisfy this criterion is uncertain, but this will need to be shown before the approach is implemented.

To explore the potential of this approach, we previously modeled the efficiency of a personalized approach to screening for prostate and breast cancers based on age and polygenic risk profile compared with the standard approach based on age alone and reported the results elsewhere. ${ }^{4}$ We found that a personalized strategy may reduce the number of people eligible for screening, whilst detecting the majority of cases identified through age-stratified standard screening, making this strategy potentially more effective than the present age-stratified breast

\footnotetext{
${ }^{1}$ Department of Public Health Genomics, PHG Foundation, Cambridge, UK; ${ }^{2}$ Department of Applied Health Research, Centre of Applied Health Research, University College London, London, UK; ${ }^{3}$ Department of Public Health and Primary Care, University of Cambridge, Cambridge, UK; ${ }^{4}$ Department of Medical Epidemiology and Biostatistics, Karolinska Institutet, Stockholm, Sweden; ${ }^{5}$ Department of Oncology, University of Cambridge, Cambridge, UK. Correspondence: Susmita Chowdhury

(susmita.chowdhury@phgfoundation.org)
} 
cancer screening. In addition, we reported that this type of personalized preventive strategy (e.g., personalized screening) has the potential to detect cancer in younger subjects at higher risk, may be more cost-effective, and may reduce harms of population screening through reduction of false-positive results, overdiagnosis, and overtreatment. Those at lower than average risk, who would otherwise be screened and exposed to investigations such as mammograms, prostate-specific antigen tests, and tissue biopsies in the age-stratified system, might avoid screening in a risk-based system because their low risk did not justify these interventions. We did not include highly penetrant variants (e.g., BRCA 1 and 2) in our model and therefore did not discuss issues related to people detected with such variants. People with such variants already follow established detection and care pathways, and the strong heritability of these alleles means that risk assessment and treatment raise different issues for individuals and family members.

There is further evidence that a collection of susceptibility variants may help to stratify the population into risk categories that can guide screening delivery programs. So and colleagues ${ }^{3}$ found, through modeling, that inclusion of genetic information of known susceptibility variants in a risk-prediction framework helps to refine risk and suggested that stratifying people into risk categories may be useful in targeted prevention and screening. Similarly, Hawken et al. ${ }^{2}$ performed analyses using both simulated and empirical population data to show significant predictive utility of genomic risk-stratified screening for colorectal cancer. Future research is likely to help in developing comprehensive risk-prediction models for breast and prostate cancers that would include the effects of environmental, lifestyle, family history, and other biomarkers and hormonal factors, alongside age and a polygenic risk profile incorporating further new variant information. This would possibly enable an even more precise individual risk assessment and therefore facilitate a truly personalized strategy for prevention of breast and prostate cancers in the population.

Personalized risk assessment using polygenic profiles can be used for primary and secondary prevention. Primary prevention could involve the use of endocrine drugs ${ }^{12,13}$ and/or modification of lifestyle to reduce weight and increase physical activity, ${ }^{14}$ although the value of the latter approach is still not clearly established. For secondary prevention, genomic information could help triage individuals to risk categories that form the basis for different types and intensities of screening, ${ }^{2}$ including an earlier screening. ${ }^{3}$ Provision of risk information may also increase the uptake of existing screening methods. ${ }^{2,15}$ There is ongoing empirical work, in a population-based pilot study, to improve risk prediction using single-nucleotide polymorphism information and to evaluate whether preventive strategies (e.g., risk-reducing counseling, weight loss, cessation of hormone therapy, referral for short-interval screening, chemoprevention, and dietary changes) can be targeted at high-risk women already undergoing breast cancer screening. ${ }^{16}$ Results from this study confirm the feasibility of determining high-risk individuals and targeting prevention strategies in these individuals. ${ }^{10}$
In the future, intervention strategies such as risk-stratified screening followed by personalized risk-reduction interventions may replace or complement the present system of population screening for complex diseases such as breast cancer. The benefits of a personalized prevention strategy may also encourage the establishment of new screening programs for other conditions such as prostate cancer. Therefore, a timely assessment of the scientific, ethical, and practical uncertainties about whether and how to provide stratified cancer screening using genetic markers is essential. To explore the wider implications of using genomic and other information in risk stratification for breast and prostate cancers, we organized two multidisciplinary expert workshops. In this article, we discuss issues that are likely to be important when considering incorporation of genetic information into population cancer prevention strategies.

\section{APPROACH}

In July 2010 and July 2011, on behalf of the multicenter Collaborative Oncological Gene-environment Study (COGS) (http://www.cogseu.org/) funded by the Seventh Framework Programme of the European Commission, the Foundation for Genomics and Population Health (PHG Foundation) in partnership with the University of Cambridge (Work Package 7 of COGS) convened two expert workshops to discuss the implications of using genetic information and risk stratification in personalized screening for breast and prostate cancers. Those attending the workshop included international leaders in oncology, breast screening services, clinical genetics, ethics, health service policy, public health, and lay representatives. The participants, who were selected for their expertise and interest in this field, were from the Karolinska Institute, the International Agency for Research on Cancer, Queen Mary University of London, Southampton University, the Institute of Cancer Research and Royal Marsden Hospital, St Mary's Hospital, University of East Anglia, the Spanish Association Against Cancer, Erasmus MC, The Times newspaper, VU University Medical Center, Cambridge University, Moncton and Sherbrooke Universities, Hughes Hall Centre for Biomedical Science in Society, Addenbrooke's Hospital, Harvard School of Public Health, Princess Anne Hospital, European Academy, Warwick University, the National Human Genome Research Institute, RAND Europe, the National Health Service cancer screening program, University of Nottingham, St George's Hospital Medical School, Jill Rogers Associates, CPO Piemonte and S Giovanni University Hospital, the PHG Foundation, Newcastle University, University of Hertfordshire, Karolinska University Hospital, and University of Leicester.

In the first workshop, the participants engaged in working groups and panel discussions to explore and identify issues related to the question "what are the issues around using risk stratification based on individual risk prediction models to target population-based screening and prevention programs?" Hexagon modeling, a systematic technique developed by Idon 
BOX 1: Themes associated with public health implications of stratified cancer screening generated in the first COGS Work Package 7 workshop

- Research and evidence

- Unresolved issues of evidence in ethnic minorities

- Principles and tools in decision making about service delivery

- Service deliveries and organizational issues

- Cost-effectiveness of risk-stratified screening

- Professional training and competences required

- Rights and ethics

- Age at which genetic testing is conducted

- Insurance and protection of individuals

- Risk perception and risk communication

- Response of individuals to positive risk assessment result

COGS, Collaborative Oncological Gene-environment Study.

BOX 2: Questions prioritized by experts participating in the first COGS Work Package 7 workshop

- What are the ranges and scenarios of service models (e.g., genetic testing at birth versus testing at the point of entering screening) that can be identified and what are the main drivers for their development (e.g., cost of genome sequencing)?

- Which feasible service delivery option of risk-based screening offers the best balance between benefit, harms, and cost?

- How can we make sure that other major ethnic groups are represented fairly in research work oriented around risk prediction?

- Can genetic testing of children for risk prediction of common cancers (e.g., breast cancer) ever be justified?

- What do we know about how people perceive risk, how best to communicate to them their risk status, and how they respond to their risk assessment results?

COGS, Collaborative Oncological Gene-environment Study.

(Pitlochry, Scotland, UK), ${ }^{17}$ was used to brainstorm, capture, and prioritize the expert opinions of the group and recorded 87 viewpoints offered by participants under 11 broad themes (Box 1).

These themes were then discussed in small groups, and several questions were generated. The delegates finally voted for and prioritized five key questions relevant to the original open question (Box 2). The prioritized questions encompassed as fully as possible the viewpoints discussed and represented areas for development before the next workshop.
At the second workshop, reports on various service delivery approaches, the age at which genetic sampling should be offered, and a report on risk perception, communication, and response were presented to and discussed by a similar group of multidisciplinary experts. Discussions in the second COGS workshop mainly centered on service planning, competences, and communication issues. The various issues, related to public health implications of incorporating genomic information in breast and prostate cancer screening, identified through the first and second COGS workshops are outlined in Figure 1.

In the second COGS workshop, issues related to the use of risk-stratified screening for preventing breast and prostate cancers were identified in six areas: the need for evidence of benefits and harms; complexities in using genetic information; when and how to do a risk assessment; organization and delivery of a personalized screening program; ethical, legal, and social issues; and issues of public engagement and understanding. These themes, which were further developed in Work Package 7 of COGS, are discussed in this article.

\section{THE NEED FOR EVIDENCE}

Evaluation of stratified screening requires answers to two main questions: how suitable for use is the risk-prediction model, and what is the effectiveness and cost-effectiveness of stratified screening versus conventional age-stratified screening?

Evidence related to components of risk assessment includes analytic and clinical validity of genotyping and phenotyping elements. The evaluation of these components of a risk model requires evidence that average risk is accurately estimated (calibration) and that the model ranks an individual's risk in the correct order, accurately labeling people as to their degree of risk in relation to that of the population as a whole (discrimination). There is evidence through modeling that inclusion of risk information from common susceptibility variants to established risk models help to refine risk,$^{10}$ improve performance of risk models, ${ }^{18,19}$ and may lead to better discrimination. ${ }^{3}$ Moreover, there is analysis of population data that shows significant predictive utility of genomic risk-stratified colorectal cancer screening. ${ }^{2}$ Modeling also shows that personalized screening strategy based on age and polygenic risk may potentially reduce the number of people eligible for screening, detecting the majority of the cancers identified through an age-stratified screening program (including those in younger people), or, alternatively, may detect a greater number of cases if the same number of individuals is screened, as in the age-based screening of breast cancer in the United Kingdom. ${ }^{4}$ If fewer individuals are screened, there may be a reduction in the potential harms associated with screening. However, this modeling does not estimate the expected number of cases detected following a screening program that would depend on the screening program sensitivity, which in turn depends on interscreening interval, screening test sensitivity, etc. ${ }^{4}$ If uptake or increased uptake of screening among those considered to be at high risk is the goal of risk-stratified screening, it is essential to know the point at which an offer of screening, or increased uptake does 


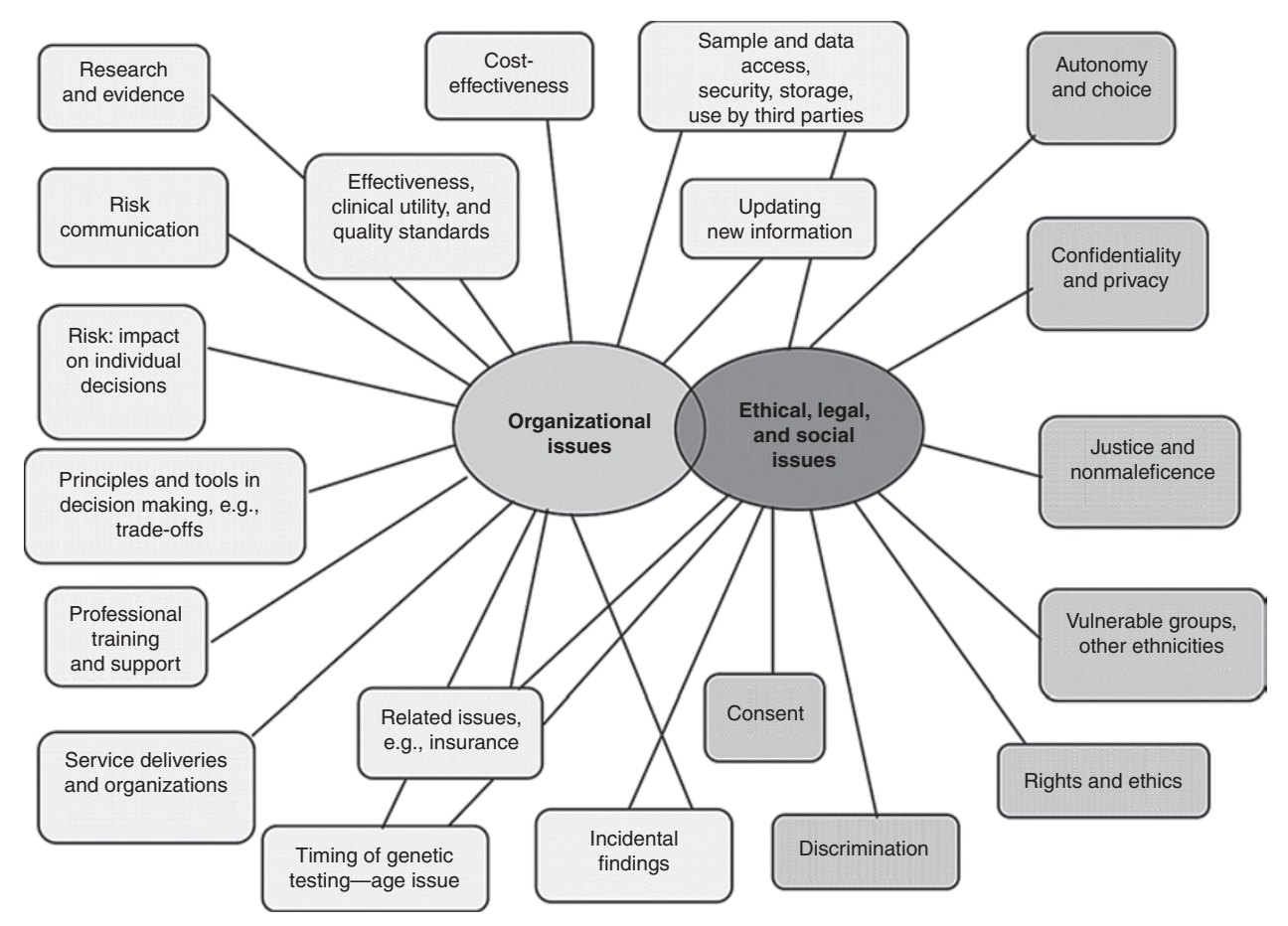

Figure 1 Issues related to public health implications of incorporating genomics in screening, explored through COGS WP7 workshops. COGS WP7, Collaborative Oncological Gene-environment Study Work Package 7.

more harm than good. There are uncertainties such as how test sensitivity will compare between younger and older subjects at the same absolute risk; the complex interaction between disease risk, age, tumor subtype, behavior, and natural history of the cancer; and whether the optimal threshold risk for one population is applicable for other populations with different cancer incidence. Therefore, although modeling can provide estimates of benefit and harm, evidence from empirical data is required to decide whether the true benefits of screening outweigh the true harms. For evaluation of the screening elements of the program, benefits in terms of reduction in morbidity and mortality must be weighed against the harms or costs, including complications of clinical investigations, anxiety over abnormal results, overdiagnosis, and even treatment of false-positive results. This is true for both age-stratified and risk-based screening. However, in risk-based screening, the evidence for each population stratum must be assessed in order to reach an aggregate value for the population as a whole.

For both sets of evidence, assumptions on effectiveness and cost-effectiveness based on results of modeling must be backed by evidence of scientific validity and clinical utility from systematic empirical research, such as pilot studies or clinical trials. Large-scale studies like COGS, with over 200,000 participants, use knowledge of genetic susceptibility from genome-wide association studies and its interaction with environmental/lifestyle factors for a more accurate individual risk prediction. KARMA (Karolinska Mammography Project for Risk Prediction of Breast Cancer) (http://karmastudy. org/) is a breast cancer cohort study involving 100,000 people in Sweden. In addition, other ongoing studies are assessing utility, feasibility, and acceptability of risk stratification, and intervention strategies for those at high risk. Early results from PROCAS (Predicting the Risk of Cancer At Screening), the largest study of its kind in the United Kingdom, indicate the feasibility of determining breast cancer risk using genomic and other information and of offering risk-reduction interventions to high-risk individuals within the context of an age-stratified screening program. ${ }^{16}$ Results from these studies and other randomized trials, as well as analyses of the implications, will be invaluable when deciding whether there is a sufficient evidence base for the implementation of population prevention strategies based on individual risk.

The cost-effectiveness analysis for risk-stratified screening as compared with age-stratified screening, particularly in the absence of data on variation of cancer detection rate and response to treatment by absolute risk threshold, is quite a challenge and is not within the scope of this article. However, costutility analysis using a suitable method of economic evaluation (such as decision modeling) and probabilistic sensitivity analysis may be useful approaches in the absence of data on variation of cancer-detection rate and response to treatment by absolute risk threshold. Cost-effectiveness analysis will need to take into account not only the direct costs of screening and treatment, but also those of collecting and managing risk-based information. These analyses will vary across health systems as well as target populations, disorders, and the type of risk-stratified screening delivery. With the current modeled cost estimates of genetic profiling, personalized screening is unlikely to be costeffective as compared with age-stratified screening. However, an increase in the number of known susceptibility variants 
will result in improvement of the efficiency of the personalized screening programs. Moreover, there may be reductions in the number of individuals eligible for screening if risk-stratified screening is introduced, resulting in relatively fewer screening tests and further investigations, as well as fewer interval cancers and overdiagnosis. ${ }^{4}$ These factors, in addition to the expected decrease in the cost of genetic profiling, may result in improvements in the cost-effectiveness of personalized screening.

\section{THE USE OF GENETIC INFORMATION}

The prospect of acquiring and using personal genetic information as part of a population-based screening program gives rise to several concerns, such as discrimination and stigmatization. ${ }^{20}$ These must be fully weighed during design and pilot phases, and managed and re-evaluated within any implemented program. Genetic information is often regarded as having special status. It is present at birth and is perceived as immutable throughout life; it is closely linked with an individual's identity and may be used as an identifier (e.g., for forensic purposes). It can predict risk of disease with greater or lesser degrees of certainty; it can provide information about family members; and it may be used to discriminate unfairly between groups or even to unjustifiably stigmatize individuals..$^{20,21}$

The complexities of using genetic information within a medical context have been dealt with at length, and good practice guidance has been developed. ${ }^{22}$ Any proposed stratified screening program must consider in detail the relevance of the many concerns about genetic information, taking into account the technologies that will be used, the implications of the data generated, and its subsequent handling. Important issues will include the need for explicit consent for undertaking analysis of DNA; whether the data generated can, or should, be used for other purposes; the possibility of generating incidental findings and how these should be dealt with; whether the information is relevant for family members and, if so, whether and how it would be shared; whether the data would be stored and, if so, with what safeguards; and who might have access, including the individual, their family members, employers, insurance companies, criminal justice agencies, and researchers. All of these issues must be resolved in the program's design and implementation to the satisfaction of the public, professionals, and policy makers.

\section{RISK ASSESSMENT}

Many practical and ethical, legal, and social issues center on the application of the tool for risk assessment. The components of the tool are likely to include at a minimum: genetic and other biomarkers; a data set of personal, clinical, and family history; reproductive information; and environmental or lifestyle factors. Whereas the genetic markers will be constant and so could be analyzed at any age, nongenetic information will change over time. The screening program therefore needs to determine when both types of information will be gathered and whether data collection should be repeated. This raises a number of questions, including what should the age for genetic testing be? When should the dynamic data set be constructed and how it will be updated, if at all? When and how will the information be integrated to produce a risk assessment "score" that can be used for stratification and the setting of decision thresholds?

The genetic information could, in theory, be determined at any age. However, the workshop participants thought that it was important to examine the possibility that testing could be done in childhood, for example, as part of a newborn screening program, with risk assessment done at a later stage. We examined this issue in detail. We considered a range of issues, including how testing at an early age might compromise future autonomy, thresholds for requiring consent or assent from children and young people, and how disagreements between parents and children should be managed. Early sampling might reduce the scientific validity of the risk assessment process, if there is no further opportunity for gathering additional information closer to the time at which participants were invited for screening. Overall, taking into account the ethical, legal, and social issues that might arise, participants agreed that, although genetic testing in early life might maximize coverage and uptake, it may be preferable to wait until individuals reach adulthood before offering sampling for adult-onset conditions.

\section{THE ORGANIZATION AND DELIVERY OF PERSONALIZED SCREENING PROGRAMS}

A risk-stratified screening program will add new complexities to the health system and will need careful planning and quality control measures. Health systems with an authoritative body to oversee, monitor, and to ensure the quality of the risk-based screening program are likely to manage the introduction of stratified screening more effectively. They will be able to put in place clinical policies and care pathways supported by a reliable organizational infrastructure. In decentralized systems in which within-country variability in providing risk-based screening services may be expected, centralization of all decision making about risk-based screening through a national system may be desirable. This approach may be aspirational and possibly not feasible; however, an easily accessible national guideline may assist providers of the risk-stratified screening service. For the purpose of this article, the breast cancer screening system of a centralized health system such as that of the United Kingdom is used as an example.

We have specified three possible approaches to the management of people once the results of a stratified risk assessment are available (detailed in Appendix). There are other approaches involving more risk categories, but the ones we mention may provide a basis for initial thinking by policy makers and others (see Box 3).

The approaches involve modifying the number of lifetime screening invitations according to risk. People at higher risk are more likely to be detected with a cancer when under screening surveillance; assuming that the prognosis of cancers is the same across risk categories, those people potentially have more to gain from each round of screening. The advantages for people at lower risk, who will participate in fewer or even perhaps no screening rounds, include: less 


\section{BOX 3: Approaches to managing people after stratified risk assessment}

APPROACH 1: INTENSIFIED SCREENING AND MANAGEMENT FOR THOSE AT HIGHER-THAN-AVERAGE RISK

- This approach aims to use risk stratification to identify and offer intensified screeninga to those at higher risk. "Intensified screening and management" denotes a range of modifications to the standard approach, such as patient education, starting screening at a younger age, cessation of screening invitations at a later age, use of additional screening tests, and prophylactic treatments. The goal would be to make earlier diagnoses in those with higher rates of diagnosis of curable cancers and offer the potential for more effective earlier treatment, and so improve their prognosis.

- People whose risk was estimated below the threshold for intensified screening would be screened and managed as if they had not had individualized risk screening, following the standard screening pathway.

APPROACH 2: REDUCED INTENSITY SCREENING FOR THOSE AT LOWER-THAN-AVERAGE RISK

- This approach aims to use risk stratification to identify and offer reduced-intensity screening to those at lower risk. Reduced intensity denotes a range of modifications of standard screening that could include starting screening at a later age, use of fewer tests, and cessation of screening invitations earlier than in people at high or average risk. The goal would be to protect people to whom screening offers least advantage from its risks and harms, and to reduce costs.

- People whose risk was estimated above the threshold for reduced intensity screening would be screened and managed as if they had not had individualized risk screening.

\section{APPROACH 3: MORE FULLY STRATIFIED}

- The approach combines the first two approaches, abandoning the age-stratified approach to screening altogether. The resulting individual risk estimates would be used to assign all participants to a screening pathway based on their individual risk. Such protocols would involve moreor less-intensified screening for those at higher or lower than average risk. The present standard approach, based on age alone in the case of breast cancer screening, would be discarded altogether.

aThe approaches are presented as modifications to an existing screening program, e.g., breast mammography. If a new screening program was established, e.g., prostate cancer, then approach 1 would involve only offering screening to people at high risk. inconvenience and discomfort, less anxiety, less radiation, and perhaps a lower risk of overdiagnosis. Therefore, reducing the number of rounds in which someone at lower risk participates may be of benefit to them and to the system because the costs will be lower. For those outside the current age group who would not otherwise be screened in an age-stratified system, screening brings some concomitant harms, including extra costs and higher risks of false-positive results, overdiagnosis, and overtreatment. Therefore, depending on the type of approach to management after risk stratification, there may be implications for those inside or outside the current age range of breast screening.

New organizational features, such as personalized risk communication and targeted intervention strategies, would need to be integrated into existing screening programs. In the first instance, appropriate systems for inviting and recalling people for risk assessment and screening need to be in place. Second, there should be a standard protocol for taking consent, genetic sampling, and use of a standardized risk assessment tool to integrate the individual's genetic data with his or her environmental, lifestyle, and hormonal data. Third, the level of risk of cancer will dictate the care pathway followed, with different pathways to follow according to an agreed number of risk strata, as described above. For example, Howell et al. ${ }^{16}$ reported that their model predicts that high-risk women should be screened every 15 months, intermediate-risk women every 3 years, and those at low risk could be screened every 6 years.

For a risk-stratified screening program, the development of clear communication methods suitable for conveying genetic information to the public is essential. For example, invitation letters may contain information about the various risk categories, the benefits and harms, and what to expect at different stages of the process. When consent is required, opt-in and optout principles should also be taken into account. As with all screening programs, there will be some who do not wish to participate. The program therefore may not only need to manage these individuals and those at low or average risk but also manage those who wish to take a nonstandard route, for example, people who choose private risk assessment but seek subsequent medical support from a tax-funded health-care system. In addition, an individual's risk of developing cancer may change over time due to medical, lifestyle, or family history changes, which the program will need to accommodate.

Before the implementation of a stratified screening program using genetic information, some health professionals will require additional competences. They may need to develop proficiency in conducting a suitable risk assessment of participants. This may involve training in: the use of computer-based tools; in communicating risk scores, and limitations of screening to individuals, and in relevant ethical issues. Individuals at lower risk will need to be informed that they may still develop cancer, as is the case with screen-ineligible younger individuals who are considered to be at low risk under the current UK system. This highlights the need for clear and informative decision aids to help service users to understand a risk-stratified 
screening process. However, this may mean that we need to provide service users with information about basic genetic concepts as well.

Some workshop experts believed that genetic risk assessment should be treated like any other biometric test, with individuals managed in the way of other programs that use nongenetic tests. Nevertheless, there is a consensus that genetic information may sometimes give rise to discrimination and stigmatization, in the way that other sensitive nongenetic information such as HIV status may do, particularly if accessed by third parties. Thus, how and when genetic samples and data are acquired, stored, and used deserves particular attention.

Women at a higher risk of breast cancer may be offered various tailored intervention strategies, including intensive surveillance and a different pattern of screening tests, including mammograms or magnetic resonance imaging, specific lifestyle advice, or even prophylactic measures such as chemoprevention or surgery. However, as with all screening programs, the benefits of screening tests must outweigh the harms. In addition, appropriate referral systems are needed, with access to genetic counselors and specialists for individuals who require them.

In the context of policy change, although there may be several ways to approach the delivery of risk-stratified screening, there was consensus from experts that it might be easier to change policy in a direction that was perceived as an addition to the current screening program of the country rather than denying users access to pre-existing services. For example, in the United Kingdom, it may be preferable to screen younger women $(<47$ years) at higher risk of developing breast cancer in addition to the present age-stratified system of screening all those who are 47-73 years of age, rather than to screen only high-risk women between the ages of 35 and 79 years. Introducing stratified screening in this way may be perceived as more acceptable to both the lay public and stakeholders than removing access to pre-existing services for those who currently meet age-based eligibility criteria. However, even the approach of screening only high-risk women between 35 and 79 years of age may be appropriate if it is acceptable to the public and there is strong evidence of overall benefit. On the other hand, introducing a new screening program, such as that for prostate cancer, which may only offer risk-stratified screening, may be less controversial. The decision to implement one of the several approaches to risk-stratified screening (see Box 3) discussed previously will depend on various practical issues, including information from empirical studies comparing the approaches in relation to acceptability by the public and the stakeholders, effectiveness, cost-effectiveness, and scientific evidence including clinical utility. Other organizational issues will include where and how screening is to be offered, who will provide it, how to explain and publicize the screening program, how to coordinate care, and how to manage long-term follow-up.

\section{ETHICAL, LEGAL, AND SOCIAL ISSUES}

The prospect of personalized screening using genetic information raises questions for individuals and for society. Perhaps the greatest challenge is to enable individuals to make autonomous and informed decisions about participation without overloading them with complex information. ${ }^{23}$ Relevant information will include the potential risks and benefits, grounds for inclusion or exclusion from screening, and subsequent referral and treatment. Some of the risks and benefits might be hard to gauge, such as the extent to which those with positive results in risk assessment might show fatalism and the extent to which those with lower genetic risk scores might indulge in more unhealthy behaviors or lifestyles. ${ }^{24}$ Identifying adverse gene variants in otherwise healthy individuals might be associated with longer-lasting psychological impact, and communicating these risks in a way that can be understood by everybody may be a considerable challenge. ${ }^{25}$

In the case of risk-based screening, the call for screening may be interpreted by third parties such as insurers and employers as a proxy for elevated risk status. Underwriters may use this information as a basis for setting higher insurance premiums. In some countries, legislation or moratoria exist that exclude the results of predictive genetic testing from insurers' consideration except in specified circumstances. ${ }^{26}$ The moratoria are reviewed periodically, and because of their temporary nature, there are concerns that they do not provide sufficient protection against discrimination.

The introduction of personalized screening programs could also be seen as undermining the principles of solidarity and fairness on which current screening programs that offer universal coverage are based. ${ }^{27}$ Moreover, the complexities inherent in personalized assessment could lead to a lack of engagement and uptake among people from certain ethnic ${ }^{28}$ and lower socioeconomic groups. ${ }^{29}$ Therefore, active communication strategies and quality assurance processes will be needed to mitigate any exacerbation of existing inequalities. Issues of distributive justice may become less pressing once genetic variants become more widely used in multiple preventive programs.

\section{PUBLIC ENGAGEMENT AND UNDERSTANDING}

Although the public's understanding of genetics differs from that of health-care professionals and other experts, ${ }^{30,31}$ lay individuals generally support the use of genetic information and genetic testing ${ }^{32}$ to improve the prevention, diagnosis, and treatment of disease. ${ }^{33}$ Tailored and personalized risk communication may increase lay knowledge and modify risk perception; ${ }^{15,34}$ therefore, health professionals may need training so that they can convey the most important risks and benefits of screening in a way that aids comprehension.

There is evidence in the literature of increased uptake of screening tests such as mammography after an intervention of personalized risk communication (e.g., of their individualized risk status) as compared with general risk communication (e.g., of population risk estimates or general information). ${ }^{15,35}$ In studies in which categorized risk score (e.g., high, medium, and low risk) was communicated, such increase in uptake seemed more pronounced among individuals who were told that they were at high risk. ${ }^{15}$ However, the extent to which the medium-risk group experienced increased uptake of screening 
is not clear. Such assessments would be important for decision making by policy makers, because a two-category approach (i.e., high and low risk) to risk assessment might be more efficient if the primary goal is to increase screening uptake in those at high risk. Nevertheless, there is a lack of empirical evidence about public opinions or preferences with regard to specifically risk-stratified screening, including whether people wish to know their individual risks of developing cancer or whether knowledge of risk influences participation in screening programs or behavior change. Further research is needed to determine how risk-stratified screening might be implemented. Important questions include clarifying the best way of providing information, identifying the possible needs of different ethnic or religious groups, and sources of possible bias including the trustworthiness and reliability of those delivering risk estimation and screening.

\section{Conclusion}

It cannot be emphasized enough that further empirical research is needed to provide evidence for scientific validity and clinical utility before risk-stratified screening programs can be implemented. Nevertheless, in the future, new knowledge about genetic susceptibility as well as technical advances and improved routes of access are likely to propel personalized prevention into health care. This signals future change and the adaptation of public health policies and prevention programs. Therefore, the timely analysis of the wider implications of using genomic information for risk-stratified screening in the context of cancer prevention is important. This article has highlighted some key challenges and identified a number of unresolved issues, including the age at which genetic testing should take place, the organizational complexities of service delivery, the potential for discrimination by third parties, risk communication, and the acceptability of this strategy to the public. Despite these and other uncertainties, we conclude that health professionals and stakeholders should be encouraged to embrace the new wave of genomics in preventive medicine and prepare for a more personalized health-care approach that may predict and prevent diseases more effectively.

\section{APPENDIX: DETAILS OF APPROACHES TO MANAGEMENT AFTER RISK STRATIFICATION Approach 1: Intensified screening and management for those at higher-than-average risk}

The intensification of prevention could take several forms. Using breast screening as an example, the following methods of intensification exist:

\section{Modifications to screening}

- Enhanced information to promote informed consent: Women at higher risk are likely to gain more from participating in the standard population screening program. This additional advantage could be explained in specific information and publicity, alongside the risks and disbenefits, targeted to those at higher risk and reinforced through clinical counseling provided as part of primary care or in a stand-alone screening service.

- Increasing the lifetime screening period: Screening of women at higher risk could start at an earlier age and/ or end at a later one, providing extra opportunities for tumor detection and conferring modestly increased risks. Because the probability of finding a tumor outside the usual screening age group is higher in these women, this might improve the effectiveness and cost-effectiveness of the intervention compared with women at normal risk. Further analysis would be necessary to confirm this and set appropriate age limits. Increasing the lifetime number of mammograms would inevitably cause increased radiation exposure, which itself could be carcinogenic. The balance of benefits of earlier detection in some women would need to be balanced against the risks of such exposure.

- Additional or alternative screening tests: For example, magnetic resonance imaging may be a more sensitive means of detecting breast cancers that, although not suitable as a replacement for X-ray mammography in population screening, might be useful as an adjunct in high-risk women.

Variations in preventive interventions for those at higherthan-average risk

- Lifestyle modification: Some lifestyle factors such as body mass index and diet affect the risk of breast cancer. More intensive efforts to alter lifestyle would be of value to women at higher risk, and could be supported by health services.

- Chemoprevention: The selective estrogen-receptor modulators tamoxifen and raloxifene are sometimes offered to high-risk women to reduce the risk of the development of breast cancer. Better individualized risk prediction would enable better targeted chemoprophylaxis as an adjunct to screening.

Chemoprevention adds costs, medicalizes people, and brings a different balance of harms and benefits. Lifelong management of chemoprophylaxis will involve dealing with drug interactions, long-term side effects, and circumstances in which the development of comorbidity indicates that chemoprophylaxis should cease.

- Prophylactic surgery: For those at very high risk, other options such as prophylactic mastectomy could also be offered. Although this carries the risks of surgery and potentially a reduced quality of life, some women may prefer this to less invasive options on the grounds that it minimizes the risks of developing a primary or secondary cancer.

\section{Approach 2: Reduced intensity screening and management for those at lower-than-average risk}

Under this approach, screening intensity could be reduced by adjusting some of the elements intensified in approach 1, but in the opposite way. Screening could start later, finish earlier or occur less often; information on the relative lack of advantage of 
screening to those at lower risk could be provided. In people at the lowest risk, the disadvantages of screening may outweigh its benefits to the extent that that they could be advised to avoid it altogether. If the cost-effectiveness of screening low-risk people was unacceptably low, then policy makers would need to consider whether to offer it at all. These modifications to the standard approach would also need evaluation before their widespread introduction.

\section{Approach 3: More fully stratified}

This approach is the most finessed of the three, maximizing the potential of stratification to tailor closely the offer of screening to the risk faced by each individual. It would also entail detailed consideration of which elements of screening and management should be adjusted, and in what way, to ensure maximum clinical utility within each risk cohort. Once again, these modifications would also need evaluation before their introduction.

\section{ACKNOWLEDGMENTS}

This work is part of the Collaborative Oncological Gene-environment Study (COGS) (http://www. cogseu.org), funded by the Seventh Framework Programme of the European Commission under grant agreement 223175 (HEALTH-F2-2009-223175). G.L. is supported by a postdoctoral fellowship awarded by the National Institute for Health Research. N.P. is supported by Cancer Research UK Clinician Scientist Fellowship. We gratefully acknowledge the participants in the two COGS International Workshops for their valuable contributions (listed in alphabetical order): Yvonne Brandberg, professor, Department of Oncology-Pathology, Karolinska Institute, Stockholm, Sweden; Graham Byrnes, head of biostatistics, International Agency for Research on Cancer, Lyon, France; Stephen Duffy, professor of cancer screening, Wolfson Institute of Preventive Medicine, Queen Mary University of London, UK; Diana Eccles, professor of cancer genetics, Southampton University, Wessex Clinical Genetics Service, Princess Anne Hospital, Southampton, UK; Rosalind Eeles, professor of oncogenetics, Institute of Cancer Research \& Royal Marsden Hospital, Sutton, Surrey, UK; Gareth Evans, consultant in genetic medicine, Regional Genetic Service, St Mary's Hospital, Manchester, UK; Richard Fordham, director, health economics, Faculty of Health, University of East Anglia, Norwich, Norfolk, UK; Ana Fernandez-Marcos, head of Studies \& Institutional Affairs, Spanish Association Against Cancer, Madrid, Spain; Richard Fordham, health economist, Faculty of Health, University of East Anglia, UK; Eveline Heijnsdijk, Department of Public Health, Erasmus MC, Rotterdam, The Netherlands; Mark Henderson, science editor, The Times newspaper, London, UK; Lidewij Henneman, researcher, social sciences, VU University Medical Center, Amsterdam, The Netherlands; Christine Hill, specialist registrar in public health, Institute of Public Health, Cambridge University, UK; Jalila Jbilou, associate professor, Moncton and Sherbrooke Universities, Moncton, Canada; Stephen John, Lecturer, Department of History and Philosophy of Science, Cambridge University, UK; Marie Keane, health-care worker, Addenbrooke's Hospital, Cambridge, UK; Peter Kraft, associate professor of epidemiology, Harvard School of Public Health, Boston, MA; Anneke Lucassen, professor of clinical genetics, Wessex Clinical Genetics Service, Princess Anne Hospital, Southampton, UK; Deborah Mascalzoni, senior scientist, European Academy, Bolzano, Italy; Kenneth Muir, senior European Union scientist, Health Sciences Research Institute, Warwick Medical School, Warwick University, UK; Colleen McBride, chief senior investigator, National Human Genome Research Institute, Bethesda, MD; Ellen Nolte, director, Health and Healthcare Policy Program, RAND Europe, Cambridge, UK; Julietta Patnick, director, National Health Service Cancer Screening Program, Sheffield, UK; Nadeem Qureshi, clinical professor of primary care, The University of Nottingham, UK; Imran Rafi, General Practitioner, senior lecturer of primary care education, St George's Hospital Medical School, London, UK; Jill Rogers, Jill Rogers Associates, Cottenham, Cambridge, UK; Nereo Segnan, head, Department of Cancer Screening and Unit of Cancer Epidemiology, CPO Piemonte and S Giovanni University Hospital, Turin, Italy; Jean Sinclair, research nurse, Medical Research Council Epidemiology Unit, Institute of Metabolic Science, Addenbrooke's Hospital, Cambridge, UK; Ros Skinner, senior fellow, PHG Foundation, Cambridge, UK; Hilary Thomas, professor of health care research, Centre for Research in Primary \& Community Care, University of Hertfordshire, UK; Sven Tornberg, director, Stockholm Screening Programs, Karolinska University Hospital, Stockholm, Sweden; Susan Wallace, lecturer in population and public health sciences, Department of Health Sciences, University of Leicester, UK. Funding was provided to Jalila Jbilou by the CIHR Team in Familial Risks of Breast Cancer Grant (CRN-8752-1).

\section{DISCLOSURE}

The authors declare no conflict of interest.

\section{REFERENCES}

1. Willard HF, Ginsburg GS. Genomic and Personalized Medicine. Academic Press: San Diego, CA, 2008.

2. Hawken SJ, Greenwood CM, Hudson TJ, et al. The utility and predictive value of combinations of low penetrance genes for screening and risk prediction of colorectal cancer. Hum Genet 2010;128:89-101.

3. So HC, Kwan JS, Cherny SS, Sham PC. Risk prediction of complex diseases from family history and known susceptibility loci, with applications for cancer screening. Am J Hum Genet 2011;88:548-565.

4. Pashayan N, Duffy SW, Chowdhury S, et al. Polygenic susceptibility to prostate and breast cancer: implications for personalised screening. $\mathrm{Br} J$ Cancer 2011;104:1656-1663.

5. Chung CC, Magalhaes WC, Gonzalez-Bosquet J, Chanock SJ. Genome-wide association studies in cancer-current and future directions. Carcinogenesis 2010;31:111-120.

6. Maher B. Personal genomes: The case of the missing heritability. Nature 2008;456:18-21.

7. Evans DG, Warwick J, Astley SM, et al. Assessing individual breast cancer risk within the U.K. National Health Service Breast Screening Program: a new paradigm for cancer prevention. Cancer Prev Res (Phila) 2012;5:943-951.

8. Mealiffe ME, Stokowski RP, Rhees BK, Prentice RL, Pettinger M, Hinds DA Assessment of clinical validity of a breast cancer risk model combining genetic and clinical information. J Nat/ Cancer Inst 2010;102:1618-1627.

9. Wacholder S, Hartge P, Prentice R, et al. Performance of common genetic variants in breast-cancer risk models. N Engl J Med 2010;362:986-993.

10. Gail MH. Discriminatory accuracy from single-nucleotide polymorphisms in models to predict breast cancer risk. J Natl Cancer Inst 2008;100:10371041.

11. Janssens AC, van Duijn CM. Genome-based prediction of common diseases: advances and prospects. Hum Mol Genet 2008;17(R2):R166-R173.

12. Cummings SR, Tice JA, Bauer $S$, et al. Prevention of breast cancer in postmenopausal women: approaches to estimating and reducing risk. J Natl Cancer Inst 2009;101:384-398. 
13. Cuzick J, DeCensi A, Arun B, et al. Preventive therapy for breast cancer: a consensus statement. Lancet Oncol 2011;12:496-503.

14. Teras LR, Goodman M, Patel AV, Diver WR, Flanders WD, Feigelson HS. Weight loss and postmenopausal breast cancer in a prospective cohort of overweight and obese US women. Cancer Causes Control 2011:22:573-579.

15. Edwards AG, Evans R, Dundon J, Haigh S, Hood K, Elwyn GJ. Personalised risk communication for informed decision making about taking screening tests. Cochrane Database Syst Rev 2006:CD001865.

16. Howell A, Astley S, Warwick J, et al. Prevention of breast cancer in the context of a national breast screening programme. J Intern Med 2012;271:321-330.

17. Tayko PRM, Soponkij U. The power of hexagon as an organization development process. AU-GSB e-Journal 2011:4.

18. Pharoah PD, Antoniou AC, Easton DF, Ponder BA. Polygenes, risk prediction and targeted prevention of breast cancer. N Engl J Med 2008;358:27962803.

19. Pharoah PD, Antoniou A, Bobrow M, Zimmern RL, Easton DF, Ponder BA Polygenic susceptibility to breast cancer and implications for prevention. Nat Genet 2002;31:33-36.

20. Geelen E, Horstman K, Marcelis CL, Doevendans PA, Van Hoyweghen I. Unravelling fears of genetic discrimination: an exploratory study of Dutch HCM families in an era of genetic non-discrimination acts. Eur J Hum Genet 2012;20:1018-1023.

21. Human Genetics Commission Inside Information. Balancing interests in the use of personal genetic data: a report by the Human Genetics Commission. 2002.

22. Royal College of Physicians, Royal College of Pathologists, British Society for Human Genetics. Consent and confidentiality in clinical genetic practice: Guidence on genetic testing and sharing genetic information. 2011. Report of the Joint Committee on Medical Genetics.

23. Lunshof JE, Chadwick R, Vorhaus DB, Church GM. From genetic privacy to open consent. Nat Rev Genet 2008;9:406-411.

24. Senior $V$, Marteau TM, Peters TJ. Will genetic testing for predisposition for disease result in fatalism? A qualitative study of parents responses to neonatal screening for familial hypercholesterolaemia. Soc Sci Med 1999;48:1857-1860.
25. Bunnik EM, Schermer MH, Janssens AC. Personal genome testing: test characteristics to clarify the discourse on ethical, legal and societal issues. BMC Med Ethics 2011;12:11.

26. UK HM Government, Association of British Insurers. Concordat and moratorium on genetics and insurance. 2011.

27. Buyx A, Prainsack B. Lifestyle-related diseases and individual responsibility through the prism of solidarity. Clinical Ethics 2012;7:79-85.

28. Chiu LF. Inequalities of access to cancer screening: a literature review. NHS Cancer Screening Programmes: UK. Cancer Screening Series No 1, 2003.

29. Moser K, Patnick J, Beral V. Inequalities in reported use of breast and cervica screening in Great Britain: analysis of cross sectional survey data. BMJ 2009;338:b2025.

30. Lea DH, Kaphingst KA, Bowen D, Lipkus I, Hadley DW. Communicating genetic and genomic information: health literacy and numeracy considerations. Public Health Genomics 2011;14:279-289.

31. Bottorff JL, Richardson C, Balneaves LG, et al. Unraveling women's perceptions of risk for breast cancer. Health Educ Res 2004;19:469-475.

32. Bruno M, Digennaro M, Tommasi S, et al. Attitude towards genetic testing for breast cancer susceptibility: a comparison of affected and unaffected women. Eur J Cancer Care (Engl) 2010;19:360-368.

33. Etchegary $\mathrm{H}$, Cappelli $\mathrm{M}$, Potter $\mathrm{B}$, et al. Attitude and knowledge about genetics and genetic testing. Public Health Genomics 2010;13:80-88.

34. Albada A, Ausems MG, Bensing JM, van Dulmen S. Tailored information about cancer risk and screening: a systematic review. Patient Educ Couns 2009:77:155-171.

35. Noar SM, Benac CN, Harris MS. Does tailoring matter? Meta-analytic review of tailored print health behavior change interventions. Psychol Bull 2007:133:673-693

(c) (i) @) This work is licensed under a Creative
Derivative Works 3.0 License. To view a copy of this license,
visit http://creativecommons.org/licenses/by-nc-nd/3.0/

